\title{
Therapeutic angiogenesis in the ischemic canine heart induced by myocardial injection of naked complementary DNA plasmid encoding hepatocyte growth factor
}

Toshihiro Funatsu, MDa

Yoshiki Sawa, MDa

Shigeaki Ohtake, MD

Toshiki Takahashi, MD ${ }^{a}$

Goro Matsumiya, MDa

Nariaki Matsuura, MD ${ }^{c}$

Toshikazu Nakamura, $\mathrm{MD}^{\mathrm{b}}$

Hikaru Matsuda, MD
From the Division of Cardiovascular Surgery, ${ }^{a}$ Department of Surgery E1, and the Division of Biochemistry, ${ }^{\mathrm{b}}$ Department of Oncology, Biomedical Research Center B7, Osaka University Graduate School of Medicine, and the Department of Pathology, School of Allied Health Science, Faculty of Medicine, Osaka University, Osaka, Japan.

Received for publication Sept 10, 2001; revisions requested Oct 10, 2001; revisions received Jan 22, 2002; accepted for publication Feb 5, 2002.

Address for reprints: Yoshiki Sawa, MD, Division of Cardiovascular Surgery, Department of Surgery, Osaka University Graduate School of Medicine E1, 2-2 Yamadaoka, Suita, Osaka 565-0871, Japan (E-mail: sawa@surg1.med.osaka-u.ac.jp).

J Thorac Cardiovasc Surg 2002;124: 1099-105

Copyright () 2002 by The American Association for Thoracic Surgery

0022-5223/2002 $\$ 35.00+0 \quad \mathbf{1 2 / 1 / 1 2 3 8 0 9}$

doi:10.1067/mtc.2002.123809
Objective: We investigated the efficacy of directly injecting a plasmid with complementary DNA encoding human hepatocyte growth factor into ischemic canine myocardium to induce angiogenesis.

Methods: Four weeks after ligation of the left anterior descending coronary artery, $125 \mu \mathrm{g}$ of a complementary DNA plasmid encoding the gene for either hepatocyte growth factor $(\mathrm{n}=8)$ or LacZ (transfection control group, $\mathrm{n}=8$ ) was injected directly into the myocardium at the border between the normal tissue and the infarction. Eight other dogs were used as a sham control group. Regional thickening fraction, which indicated contractile function, and blood flow in the normal (circumflex branch territory) and ischemic areas were evaluated under dobutamine administration just before and 4 weeks after transfection. The animals were killed, and capillary numbers in both areas were assessed. These data in the ischemic area were evaluated as the percentage of those in the normal.

Results: The number of myocardial capillaries in the ischemic area was successfully increased to approximately $140 \%$ of usual in the hepatocyte growth factor group, whereas no change was observed in the other groups $(P=.0017$ by analysis of variance). Furthermore, regional thickening fraction and blood flow in the ischemic area, which had deteriorated after coronary ligation, showed significant improvement in the hepatocyte growth factor group relative to the other groups (thickening fraction $P<.0001$ by analysis of variance, blood flow $P=.0005$ by analysis of variance).

Conclusions: These results support the efficacy of the direct injection of plasmid complementary DNA encoding human hepatocyte growth factor to induce therapeutic angiogenesis in the ischemic myocardium.

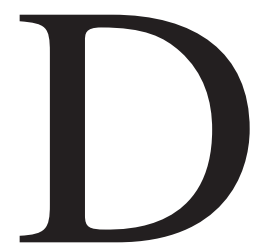

espite major advances in both surgical and catheter revascularization for ischemic heart disease, some patients cannot benefit from these treatments because of diffuse coronary artery lesions, repeated previous interventions, or low tolerance for surgical stress. In such cases new vasculature that is induced by some agent, therapeutic angiogenesis, ${ }^{1}$ might supply blood flow to the ischemic lesion and thus bring benefits comparable to those seen with either catheter angioplasty or surgical grafting.

Recent studies have shown that some growth factors have a potent ability to 
induce angiogenesis. ${ }^{2-9}$ One of the most powerful of these, vascular endothelial growth factor (VEGF), is reported to promote angiogenesis in the limbs and heart when administered as either recombinant protein or complementary DNA (cDNA) in ischemic animal models. ${ }^{2-7}$ A clinical trial with VEGF is underway. ${ }^{10,11}$

Hepatocyte growth factor (HGF), another potential therapeutic agent, was originally identified and characterized as a potent mitogen for hepatocytes. ${ }^{12}$ It has since become clear that HGF is a mesenchyme-derived pleiotropic factor that regulates cell growth, cell motility, and the morphogenesis of various cell types. ${ }^{13} \mathrm{HGF}$ is also known to stimulate endothelial cell growth without inducing the replication of vascular smooth muscle cells; thus HGF has a potent ability to induce angiogenesis. ${ }^{14-17}$ Moreover, c-Met, the specific receptor of HGF, is upwardly regulated in ischemic myocardium. ${ }^{18,19}$ With this body of evidence as a working basis, the ability of human recombinant HGF to induce therapeutic angiogenesis in the rabbit ischemic hind limb was tested and demonstrated. ${ }^{20}$ However, the efficacy of HGF to stimulate angiogenesis in the ischemic heart has not yet been well examined. In particular, investigations aimed at clinical applications are lacking.

Virus-mediated gene delivery into organs in vivo has advantages in terms of efficiency. However, several problems remain to be resolved before this method can be applied to the clinical setting, especially with respect to safety for systemic organs. In contrast, localized transfection of naked cDNA as a plasmid, without vectors, is considered to be safer but has a low efficiency of transfection. In this study we investigated the feasibility of direct injection of naked cDNA encoding HGF as a therapeutic angiogenic agent in an ischemic canine heart model.

\section{Material and Methods}

\section{Left Coronary Artery Ligation Model}

Twenty-four beagles of both sexes weighing 8 to $10 \mathrm{~kg}$ were used in these experiments. All animals received humane care in compliance with the "Principles of Laboratory Animal Care" formulated by the National Society for Medical Research and the "Guide for the Care and Use of Laboratory Animals" prepared by the Institute of Laboratory Animal Resources, National Research Council, and published by the National Academy Press, revised 1996. After intramuscular sedation with ketamine $(10 \mathrm{mg} / \mathrm{kg})$ and intravenous administration of sodium pentobarbital $(10 \mathrm{mg} / \mathrm{kg})$, the dogs were intubated endotracheally under mechanical ventilation, and anesthesia was maintained with $1 \%$ sevoflurane inhalation. Minimal left thoracotomy was performed through the fourth intercostal space, and a pericardiotomy was made. The left anterior descending coronary artery was carefully dissected and ligated just distal of the first diagonal branch. The pericardium and chest were closed in layers, and the animals were allowed to recover.

\section{Target Region of Gene Transfection}

Four weeks after the coronary ligation, thoracotomy through the left fifth intercostal space was performed on the animals with the same anesthesia protocol described previously. In all animals infarcted myocardium caused by previous ligation was visible as a white scar along the left anterior descending coronary artery. The ischemic area targeted by gene transfection was established at the border zone between the normal and the infarcted areas by visual inspection. To confirm adequacy of the area as potentially ischemic, we used epicardial echocardiography and epicardial crystal pulsed Doppler technique to examined the attenuation of contractile function with dobutamine administration. In the following protocols of functional measurement, myocardial ischemia in the target region was induced by dobutamine administration of 20 $\mu \mathrm{g} /(\mathrm{min} \cdot \mathrm{kg})$. The normal myocardium control area was chosen from the circumflex coronary artery territory.

\section{Human Hepatocyte Growth Factor Complementary DNA Plasmid Preparation and Injection}

Human HGF (hHGF) cDNA was inserted into the NotI site of the pUC-SR $\alpha$ expression vector. After assessment of the regional left ventricular function and blood flow, $0.9 \mathrm{~mL}$ of plasmid solution containing $125 \mu \mathrm{g}$ cDNA was injected directly into the myocardium of the target area at six points with a $1-\mathrm{mL}$ syringe and 27-gauge needle. Sixteen dogs were divided into two groups according to the type of cDNA injected, hHGF cDNA plasmid (HGF group, $\mathrm{n}=8$ ) or LacZ cDNA plasmid (LacZ group, $\mathrm{n}=8$ ). Eight other dogs in which the left anterior descending coronary artery was ligated underwent thoracotomy but were not injected with cDNA (sham control group, $\mathrm{n}=8$ ). The injected area was marked with 5-0 polypropylene epicardial sutures. Four weeks after the cDNA injection, the animals were anesthetized, and regional cardiac function and blood flow were evaluated again. At the end of study period, the dogs were killed with an overdose intravenous injection of potassium, and the hearts were removed for further analysis.

\section{Assay of Human Hepatocyte Growth Factor}

To perform the enzyme-linked immunosorbent assay (ELISA) of hHGF in the treated myocardium, 3 dogs from each group were killed 5 days after the cDNA injection, and their hearts were excised. The hearts were dissected, and blocks of myocardium from the treated and control regions were obtained according to the previous marking suture. The level of hHGF in the myocardium was estimated with an anti-hHGF monoclonal antibody (Institute of Immunology, Tokyo, Japan). The antibody that we used in the ELISA specifically detects hHGF but not endogenous canine HGF.

\section{Assessment of Regional Cardiac Function}

Regional myocardial function was evaluated by left ventricular wall thickening fraction (TF) with the epicardial crystal pulsed Doppler technique. ${ }^{21}$ To estimate the left ventricular wall thickness, ultrasonic crystals (WT-20; Crystal Biotech, Hopkinton, Mass) were implanted into the epicardium both in the territory of circumflex coronary artery (normal area) and close to the gene injection site (ischemic area). For pressure measurement and instantaneous pressure (dp/dt) monitoring, an $8 \mathrm{~F}$ catheter-tip micromanometer (Millar Instruments, Inc, Houston, Tex) was intro- 
duced into the left ventricle through its apex. Wall thickness, left ventricular pressure, and dp/dt data were recorded and analyzed with computer software (MacLab v3.5/c; AD Instruments Pty Ltd, Castle Hill, New South Wales, Australia). After stabilization, data on each parameter were collected at rest and under stress from administration of dobutamine to cause an ischemic condition. Dobutamine administration was started at $5 \mu \mathrm{g} /(\mathrm{kg} \cdot \min )$ and raised every 10 minutes to a final dose of $20 \mu \mathrm{g} /(\mathrm{kg} \cdot \mathrm{min})$. TFs of left ventricular wall in normal and ischemic regions were calculated from the collected data as follows:

$$
\mathrm{TF}=\frac{\text { end-systolic thickness }- \text { end-diastolic thickness }}{\text { end-diastolic thickness }} \times 100 \%
$$

\section{Myocardial Blood Flow Measurement}

After the measurement of cardiac function, colored microspheres were used to evaluate myocardial blood flow in both the ischemic and normal regions. About 3 million colored microspheres (Triton Technology Inc, San Diego, Calif) suspended in $10 \mathrm{~mL}$ saline solution were injected through the left atrial appendage during 20 seconds. Reference blood sampling was started 5 seconds before the injection of the microsphere solution, and $9 \mathrm{~mL}$ blood was withdrawn at a constant rate of $6 \mathrm{~mL} / \mathrm{min}$ through a cannula inserted into the femoral artery. For the assessment 4 weeks after the cDNA injection, after the functional measurement and blood draw were completed, the animal was killed and the heart was excised. The microsphere-determined perfusion flow was measured by a spectrophotometry method. ${ }^{22}$ Myocardial samples of approximately $1 \mathrm{~g}$ each were taken from the ischemic, geneinjected territory and the normal myocardium. The myocardial samples and reference blood samples were digested overnight in $10 \mathrm{~mL}$ of $10-\mathrm{mol} / \mathrm{L}$ potassium hydroxide. The microspheres were extracted with dimethylformaldehyde, and the optical density (OD) of the dye was analyzed with a spectrophotometer (UV160A; Shimadzu Inc, Tokyo, Japan). From the OD measured, the myocardial blood flow was calculated according to the following equation:

Blood flow in tissue sample $=\frac{\text { withdrawal rate }}{\text { tissue sample weight }} \times$

$$
\frac{\text { OD of tissue sample }}{\text { OD of reference blood sample }}
$$

where blood flow is in milliliters per minute per gram, withdrawal rate is in milliliters per minute, and weight is in grams.

\section{In Vitro Histologic Assessment}

The excised hearts from all the animals were cut, and tissue specimens were obtained as transverse sections from the ischemic, cDNA-injected area and the normal area. Both myocardial samples were embedded in compound (Tissue-Tek; Sakura Finetechnical Co, Ltd, Tokyo, Japan) and frozen in liquid nitrogen. Frozen 5- $\mu \mathrm{m}$ thick sections were cut transversely from each specimen with a cryostat. To count the capillaries in the myocardium, epithelial cells were detected by staining the frozen sections immunohistologically with an antibody to von Willebrand factor with the Dako Enhanced Polymer One-step Staining kit (DAKO Japan Co Ltd, Kyoto, Japan). The peroxidase was visualized by incubation with (ng/g tissue)

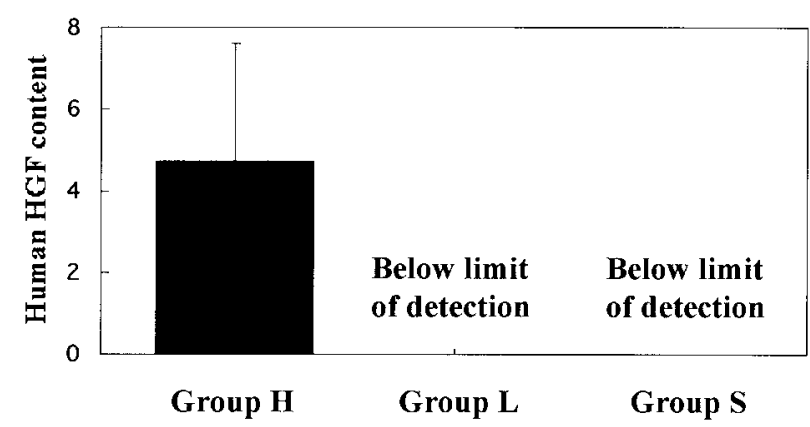

Figure 1. Comparison of hHGF protein contents in myocardium of cDNA-injected ischemic region measured 5 days after gene delivery in HGF (Group H), LacZ (Group L), and sham control (Group S) groups. Bar heights represent mean; error bars represent SD.

$\mathrm{DAB}$, followed by incubation with a DAB-enhancing solution (DAKO). Ten different fields at $200 \times$ magnification were randomly selected, and the number of capillaries in each field was counted. The average density of capillaries in the DNA-injected area was expressed as the percentage of the average capillary density in the normal area.

\section{Safety Issues}

To assess the adverse effects of this protocol, the electrocardiogram was monitored to detect arrhythmia during the entire experimental protocol, and the leukocyte count and serum hHGF levels were measured in 3 dogs from each group 1, 3, 5, 7, and 14 days after cDNA injection. The serum HGF level was assessed with the ELISA system described previously.

\section{Statistical Analysis}

All values are expressed as mean \pm SD. A 1-way analysis of variance, followed by the Bonferroni test, was used to compare the means of the three groups.

\section{Results}

Overexpression of Human Hepatocyte Growth Factor in the Myocardium after Plasmid Injection

To ensure that our method resulted in sufficient production of $\mathrm{HGF}$, the hHGF content in the myocardium samples obtained from the cDNA-injected area was determined with an ELISA assay. The result was $4.7 \pm 1.7 \mathrm{ng} / \mathrm{g}$ tissue in the HGF group, whereas hHGF was undetectable in the LacZ and sham control groups (Figure 1). Samples obtained from the normal areas in all groups contained no hHGF protein.

\section{Immunohistochemical Staining and Capillary Density}

Four weeks after the HGF transfection, the capillary density in the ischemic region was the same as in the nonischemic region in the LacZ and sham control groups, whereas a significant increase to approximately $140 \%$ of the value in the normal area was shown in the HGF group (capillary 

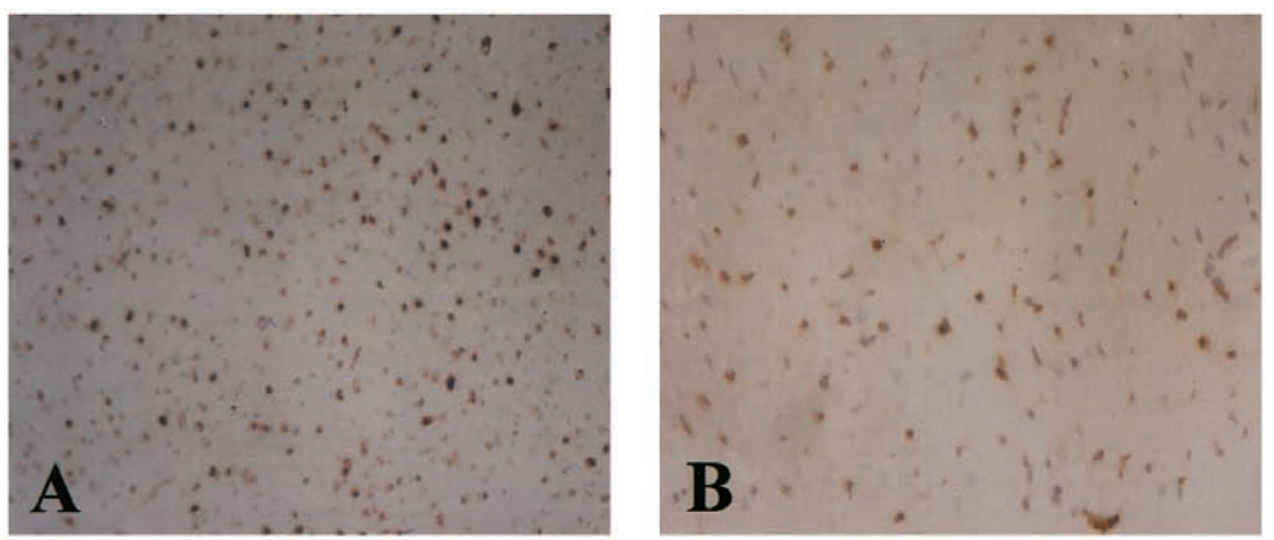

Figure 2. Representative immunohistologic staining by von Willebrand antibody of ischemic myocardium taken from cDNA-treated area 4 weeks after gene transfection (original magnification $\times 200$ ). A, Treated with cDNA encoding HGF; B, treated with cDNA encoding LacZ.

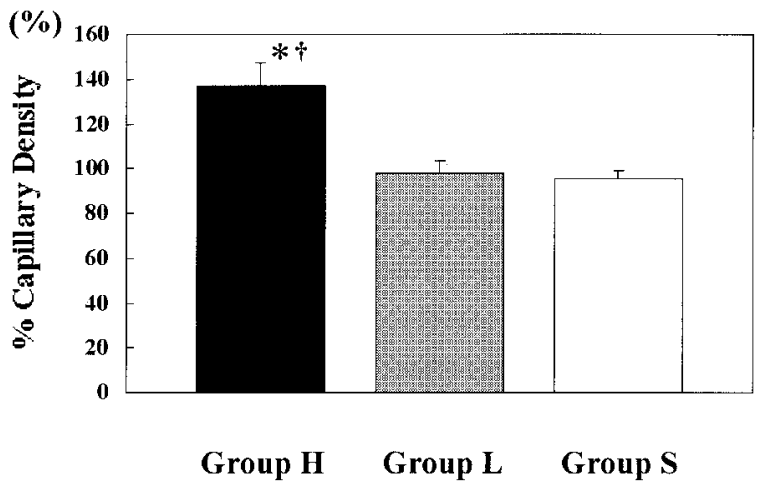

Figure 3. Comparison of capillary densities in cDNA-treated ischemic myocardium 4 weeks after gene delivery in HGF (Group H), LacZ (Group L), and sham control (Group S) groups. Bar heights represent mean; error bars represent SD. Asterisk indicates $\boldsymbol{P}=$ .0018 versus LacZ group; dagger indicates $P=.0011$ versus sham control group.

density $137.0 \% \pm 22.5 \%$ in HGF group vs $97.6 \% \pm 12.2 \%$ in LacZ group vs $95.0 \% \pm 9.1 \%$ in sham control group, $P=$ .0017 ; Figures 2 and 3).

\section{Recovery of Regional Contraction and Perfusion}

In light of these results, we analyzed the efficacy of hHGF plasmid injection with respect to the regional contractile function and blood supply. TF, as an indicator of contraction, and blood flow were measured under dobutamine administration stress to induce ischemia. Both parameters in the ischemic region were evaluated as the percentage of the same measurement in the normal area of the same heart. TF in the ischemic area, which had deteriorated as a result of the coronary ligation $33.5 \% \pm 13.7 \%$ in HGF group vs $31.7 \% \pm 7.7 \%$ in LacZ group vs $27.1 \% \pm 10.1 \%$ in sham control group), showed significant improvement 4 weeks after

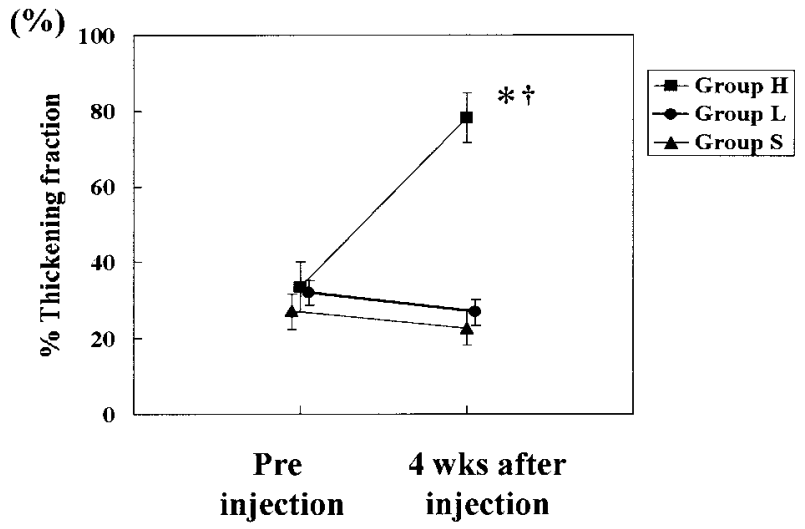

Figure 4. Comparison of wall contractile function evaluated by TF in cDNA-treated ischemic region with administration of dobutamine (20 $\mu \mathrm{g} /[\mathrm{kg} \cdot \mathrm{min}])$ in HGF (squares), LacZ (circles), and sham control (triangles) groups. Results are expressed as percentage of value in normal area. Data points represent mean; error bars represent SD. Asterisk indicates $\boldsymbol{P}<.0001$ versus LacZ group; dagger indicates $\boldsymbol{P}<.0001$ versus sham control group.

injection only in the HGF group $(78.1 \% \pm 11.2 \%$ in $\mathrm{HGF}$ group vs $26.9 \% \pm 14.5 \%$ in LacZ group vs $22.7 \% \pm 12.6 \%$ in sham control group, $P<.0001$, Figure 4$)$. Regional blood flow in the ischemic area was also restricted in all groups $(67.3 \% \pm$ $9.8 \%$ in HGF group vs $54.4 \% \pm 16.5 \%$ in LacZ group vs $62.4 \% \pm 13.0 \%$ in sham control group), and recovered significantly only in the HGF group $(104.6 \% \pm 9.3 \%$ in HGF group vs $58.0 \% \pm 20.2 \%$ in LacZ group vs $61.0 \% \pm 13.6 \%$ in sham control group, $P=.0005$; Figure 5).

\section{Safety of Direct Injection of Hepatocyte Growth}

Factor Complementary DNA Plasmid

No animals died unexpectedly during the study period. The effect of overexpressed HGF on the living body was 
evaluated with a serial leukocyte count, assessment of the serum hHGF level, and an electrocardiogram. The leukocyte count, which had risen immediately after the cDNA injection, was reduced promptly to the normal range within a week, and the changes in the leukocyte count in time were similar in the three groups (data not shown). No hHGF was detected in the serum in any group during the study period. Furthermore, no critical arrhythmias were observed during postoperative electrocardiogram monitoring, although some paroxysmal ventricular beats were observed during the direct injection of the cDNA plasmid into the myocardium.

\section{Discussion}

The potent capability of humoral growth factors, represented by VEGF and fibroblast growth factor, to induce new vessel formation has been shown in many animal models of ischemic limb and heart. ${ }^{2-9}$ Recently, in addition to these factors, HGF was also shown to have an angiogenic effect by stimulating epithelial cell growth without causing smooth muscle cell replication. ${ }^{14-17}$

In this study we successfully demonstrated that transfer of the cDNA encoding hHGF by direct injection of a plasmid resulted in increases in regional capillary density and regional blood supply in the ischemic canine heart. Furthermore, this angiogenic effect was associated with improvement in the regional contractile function during stress-induced ischemia. This is the first report to our knowledge to attest to the efficacy of the direct injection of the gene for hHGF by plasmid for the potential clinical application of angiogenesis therapy in the ischemic heart.

In our study the number of capillaries had increased 4 weeks after treatment, although the increase in large-caliber vessels was limited. The improvement of regional blood flow in the treated myocardium seemed to be more than expected from the increase of capillary density. We speculate that some endogenous factors, such as nitric oxide derived from the endothelium of newly formed capillaries, may play a role in the recovery of blood perfusion.

Several methods for gene delivery have been established, and some of them are already being applied in clinical investigations, including transcoronary or myocardial injection and transfection with a viral vector ${ }^{11}$ or a naked plasmid. ${ }^{10}$ Rosengart and colleagues ${ }^{11}$ showed successful clinical improvement in ischemic heart disease with direct myocardial injection of adenoviral-mediated cDNA encoding VEGF. On the other hand, Losordo and colleagues ${ }^{10}$ reported comparable results by direct myocardial injection of a cDNA plasmid encoding human VEGF. In our study we used direct injection of the cDNA plasmid as the gene delivery system, in the same manner as Losordo and colleagues. ${ }^{10}$ Obviously the efficiency of transfection and the subsequent protein expression resulting from direct injection of plasmid cDNA could be lower than that obtained

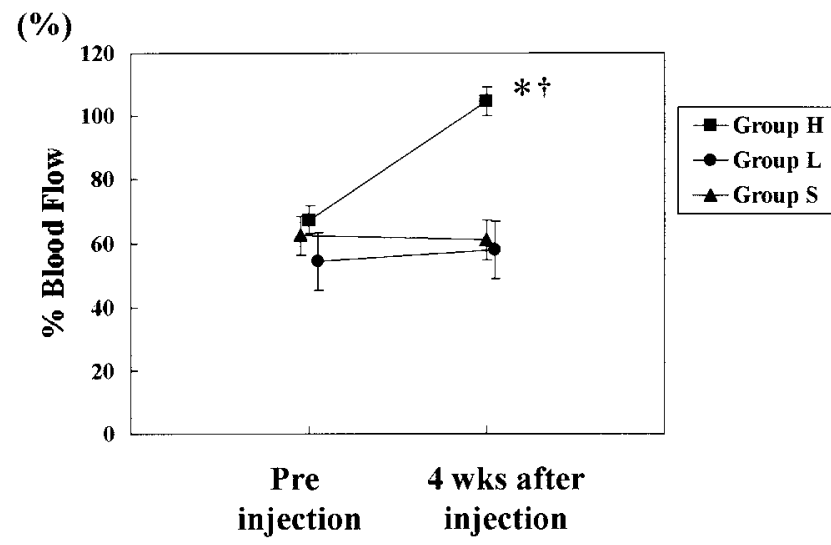

Figure 5. Comparison of blood perfusion in cDNA-treated ischemic region with administration of dobutamine $(20 \mu \mathrm{g} /[\mathrm{kg} \cdot \mathrm{min}])$ in HGF (squares), LacZ (circles), and sham control (triangles) groups. Results are expressed as percentage of value in normal area. Data points represent mean; error bars represent SD. Asterisk indicates $\boldsymbol{P}=.0004$ versus LacZ group; dagger indicates $\boldsymbol{P}=.0006$ versus sham control group.

with virus-mediated transfection. However, cDNA plasmid injection has been shown previously to yield expression of a reporter gene for longer than a month. ${ }^{23}$ Furthermore, we confirmed the expression of the cDNA that we transfected into the myocardium by plasmid injection, indicating the feasibility of this method for gene transfer.

Indeed, the concentrations of HGF protein that we detected in our myocardial samples were somewhat low compared with those obtained with the hemagglutinating virus of Japan-mediated intracoronary infusion that we reported previously. ${ }^{18}$ However, HGF, a secreted protein, can act in an autocrine-paracrine manner, ${ }^{14,24}$ and thus a lower quantity of continuously expressed protein could be sufficient to induce angiogenesis and support the subsequent recovery of regional cardiac function in vivo, as Losordo and colleagues ${ }^{10}$ have shown.

Some adverse effects from these angiogenic growth factors have been seen; the formation of tumor vessels, such as hemangiomas, may be one of the most serious problems. In a previous study, the unregulated expression of VEGF in the heart led to the formation of hemangiomalike vessels. ${ }^{6,}{ }^{25}$ In this study no tumorlike vessels were seen in the heart 4 weeks after HGF transfection. Indeed, it is to be expected that the expression of excessively high levels of HGF would result in the abnormal growth of new vessels. However, the direct injection of the cDNA plasmid, which induces the expression of relatively small amounts of protein, might not cause such adverse phenomena.

Overexpression of HGF has been shown to induce angiogenesis directly and indirectly through the activation of VEGF gene transcription. ${ }^{26}$ In its signaling pathway, HGF is upstream of VEGF; thus gene therapy with HGF is expected to 
have an angiogenic effect comparable or superior to that of VEGF, although no confirmation has yet been obtained. Several clinical trials aimed at therapeutic angiogenesis with VEGF gene transfection with adenoviral vectors ${ }^{11}$ as well as plasmids ${ }^{10}$ have been initiated, and the short-term results have been reported to be satisfactory. These successes have encouraged us to plan direct injection of a plasmid containing cDNA encoding hHGF in cases of otherwise untreatable ischemic heart disease in the near future.

There may be other advantages to using HGF in preference to other growth factors to treat the ischemic heart. For example, in addition to its angiogenic effects, HGF has other physiologic activities, such as antiapoptosis and antifibrosis. We have previously demonstrated the amelioration of ischemia-reperfusion injury and infarcted area by HGF through its antiapoptotic effect. ${ }^{18,27}$ These effects may contribute to the functional recovery of the ischemic heart. In contrast, VEGF is known to have adverse effects, such as increasing the membrane permeability, ${ }^{28}$ which may lead to myocardial edema. Further investigation is required to ascertain the therapeutic superiority of HGF or VEGF.

Although the overall clinical results from coronary artery bypass grafting and percutaneous transluminal coronary angioplasty are improving, some patients with ischemic heart disease have untreatable coronary lesions. Most of these patients have critical symptoms caused by the ischemic myocardium that may progress to ischemic cardiomyopathy. In this study we showed the efficacy of hHGF plasmid treatment, which induced neoangiogenesis in the ischemic myocardium, improving regional myocardial perfusion and contractile function. We believe that this treatment would be an invaluable option for such a patient, either combined with coronary artery bypass grafting or as a sole therapy.

There are several limitations to our study. First, dogs are known to have native collateral vessels in heart. Therefore it is possible that such native collateral formation affected the functional recovery. Second, our definition of the ischemic region was imprecise to some extent, because we performed acute coronary ligation. Indeed, the determination of ischemic myocardium induced by dobutamine administration was difficult and relatively subjective, whereas we could check the infarcted myocardium visually. Third, we did not assess the size of infarction. The effect of HGF treatment for infarcted myocardium was not assessed in this study, and further investigation is required.

In conclusion, we confirmed the efficacy of injection into the ischemic myocardium of a plasmid encoding hHGF as an angiogenic therapy. These results support the application of hHGF plasmid gene therapy to the clinical setting. The simplicity of this procedure is especially attractive for high- risk patients, either as a sole therapy or combined with conventional coronary artery bypass grafting.

We thank S. Matsumi and A. Nishimura for providing veterinary care and laboratory support and S. Kotouda of Eiko Science, Inc, for functional measurement support.

\section{References}

1. Hockel M, Schlenger K, Doctrow S, Kissel T, Vaupel P. Therapeutic angiogenesis. Arch Surg. 1993;128:423-9.

2. Mack CA, Patel SR, Schwarz EA, Zanzonico P, Hahn RT, Ilercil A, et al. Biologic bypass with the use of adenovirus-mediated gene transfer of the complementary deoxyribonucleic acid for vascular endothelial growth factor 121 improves myocardial perfusion and function in the ischemic porcine heart. $J$ Thorac Cardiovasc Surg. 1998;115:168-77.

3. Banai S, Jaklitsch MT, Shou M, Lazarous DF, Scheinowitz M, Biro S, et al. Angiogenic-induced enhancement of collateral blood flow to ischemic myocardium by vascular endothelial growth factor in dogs. Circulation. 1994;89:2183-9.

4. Magovern CJ, Mack CA, Zhang J, Hahn RT, Ko W, Isom OW, et al. Direct in vivo gene transfer to canine myocardium using a replicationdeficient adenovirus vector. Ann Thorac Surg. 1996;62:425-34.

5. Lee LY, Patel SR, Hackett NR, Mack CA, Polce DR, El-Sawy T, et al. Focal angiogen therapy using intramyocardial delivery of an adenovirus vector coding for vascular endothelial growth factor 121. Ann Thorac Surg. 2000;69:14-24.

6. Schwarz ER, Speakman MT, Patterson M, Hale SS, Isner JM, Kedes $\mathrm{LH}$, et al. Evaluation of the effects of intramyocardial injection of DNA expressing vascular endothelial growth factor (VEGF) in a myocardial infarction model in the rat: angiogenesis and angioma formation. J Am Coll Cardiol. 2000;35:1323-30.

7. Poliakova L, Kovesdi I, Wang X, Capogrossi MC, Talan M. Vascular permeability effect of adenovirus-mediated vascular endothelial growth factor gene transfer to the rabbit and rat skeletal muscle. J Thorac Cardiovasc Surg. 1999;118:339-47.

8. Shou M, Thirumurti V, Rajanayagam MA, Lazarous DF, Hodge E, Stiber JA, et al. Effect of basic fibroblast growth factor on myocardial angiogenesis in dogs with mature collateral vessels. J Am Coll Cardiol. 1997;29:1102-6.

9. Harada K, Grossman W, Friedman M, Edelman ER, Prasad PV, Keighley CS, et al. Basic fibroblast growth factor improves myocardial function in chronically ischemic porcine hearts. J Clin Invest. 1994;94:623-30.

10. Losordo DW, Vale PR, Symes JF, Dunnington CH, Esakof DD, Maysky M, et al. Gene therapy for myocardial angiogenesis. Initial clinical results with direct myocardial injection of phVEGF165 as sole therapy for myocardial ischemia. Circulation. 1998;98:2800-4.

11. Rosengart TK, Lee LY, Patel SR, Sanborn TA, Parikh M, Bergman $\mathrm{GW}$, et al. Angiogenesis gene therapy: Phase I assessment of direct intramyocardial administration of an adenovirus vector expressing VEGF121 cDNA to individuals with clinically significant severe coronary artery disease. Circulation. 1999;100:468-74.

12. Nakamura T, Nishizawa T, Hagiya M, Seki T, Shimonishi M, Sugimura A, et al. Molecular cloning and expression of human hepatocyte growth factor. Nature. 1989;342:440-3.

13. Matsumoto K, Nakamura T. Emerging multipotent aspects of hepatocyte growth factor. J Biochem. 1996;119:591-600.

14. Hayashi S, Morishita R, Higaki J, Aoki M, Moriguchi A, Kida I, et al. Autocrine-paracrine effects of over-expression hepatocyte growth factor gene on growth of endothelial cells. Biochem Biophys Res Commun. 1996;220:539-45.

15. Bussolino F, Di Renzo MF, Ziche M, Bocchietto E, Olivero M, Naldini L, et al. Hepatocyte growth factor is a potent angiogenic factor which stimulates endothelial cell motility and growth. J Cell Biol. 1992;119:629-41.

16. Silvagno F, Follenzi A, Arese M, Prat M, Giraudo E, Gaudino G, et al. In vivo activation of met tyrosine kinase by heterodimeric hepatocyte 
growth factor molecule promotes angiogenesis. Arterioscler Thromb Vasc Biol. 1995; 15:1857-65.

17. Nakamura Y, Morishita R, Higaki J, Kida I, Aoki M, Moriguchi A, et al. Hepatocyte growth factor is a novel member of the endotheliumspecific growth factors: additive stimulatory effect of hepatocyte growth factor with basic fibroblast growth factor but not with vascular endothelial growth factor. J Hypertens. 1996;14:1067-72.

18. Ueda H, Sawa Y, Matsumoto K, Kitagawa-Sakakida S, Kawahira Y, et al. Gene transfection of hepatocyte growth factor attenuates reperfusion injury in the heart. Ann Thorac Surg. 1999;67:1726-31.

19. Ono K, Matsumori A, Shioi T, Furukawa Y, Sasayama S. Enhanced expression of hepatocyte growth factor/c-Met by myocardial ischemia and reperfusion in a rat model. Circulation. 1997;95:2552-8.

20. Morishita R, Nakamura S, Hayashi S, Taniyama Y, Moriguchi A, Nagano T, et al. Therapeutic angiogenesis induced by human recombinant hepatocyte growth factor in rabbit hind limb ischemia model as cytokine supplement therapy. Hypertension. 1999;33:1379-84.

21. Zhu WX, Myers ML, Hartley CJ, Roberts R, Bolli R. Validation of a single crystal for measurement of transmural and epicardial thickening. Am J Physiol. 1986;251(5 Pt 2):H1045-55.

22. Kowallik P, Schulz R, Guth BD, Schade A, Paffhausen W, Gross R, et al. Measurement of regional myocardial blood flow with multiple colored microspheres. Circulation. 1991;83:974-82.
23. Lin H, Parmacek MS, Morle G, Bolling S, Leiden JM. Expression of recombinant genes in myocardium in vivo after direct injection of DNA. Circulation. 1990;82:2217-21.

24. Kono S, Nagaike M, Matsumoto K, Nakamura T. Marked induction of hepatocyte growth factor mRNA in intact kidney and spleen in response to injury of distant organs. Biochem Biophys Res Commun. 1992;186:991-8.

25. Lee RJ, Springer ML, Blanco-Bose WE, Shaw R, Ursell PC, Blau HM. VEGF gene delivery to myocardium: deleterious effects of unregulated expression. Circulation. 2000;102:898-901.

26. Belle EV, Witzenbichler B, Chen D, Silver M, Chang L, Schwall R, et al. Potentiated angiogenic effect of scatter factor/hepatocyte growth factor via induction of vascular endothelial growth factor: the case for paracrine amplification of angiogenesis. Circulation. 1998;97:381-90.

27. Nakamura T, Mizuno S, Matsumoto K, Sawa Y, Matsuda H, Nakamura T. Myocardial protection from ischemia/reperfusion injury by endogenous and exogenous HGF. J Clin Invest. 2000;106: 1511-9.

28. Gille J, Khalik M, Konig V, Kaufmann R. Hepatocyte growth factor/ scatter factor (HGF/SF) induces vascular permeability factor (VPF/ VEGF) expression by cultured keratinocytes. J Invest Dermatol. 1998; 111:1160-5. 